\title{
Non-isothermal degradation kinetics of filled with rise husk ash polypropene composites
}

\author{
S. Ch. Turmanova ${ }^{*}$, S. D. Genieva ${ }^{2}$, A. S. Dimitrova ${ }^{1}$, L. T. Vlaev ${ }^{2}$ \\ ${ }^{1}$ Department of Materials Science, Assen Zlatarov University, 8010 Bourgas, Bulgaria \\ ${ }^{2}$ Department of Physical Chemistry, Assen Zlatarov University, 8010 Bourgas, Bulgaria
}

Received 16 November 2007; accepted in revised form 10 January 2008

\begin{abstract}
The thermal stability and kinetics of non-isothermal degradation of polypropene and polypropene composites filled with 20 mass\% vigorously grounded and mixed raw rice husks (RRH), black rice husks ash (BRHA), white rice husks ash (WRHA) and Aerosil Degussa (AR) were studied. The calculation procedures of Coats - Redfern, Madhysudanan et al., Tang et al., Wanjun et al. and 27 model kinetic equations were used. The kinetics of thermal degradation were found to be best described by kinetic equations of $n$-th order $\left(F_{n}\right.$ mechanism). The kinetic parameters $E, A, \Delta S^{\neq}, \Delta H^{\neq}$and $\Delta G^{\neq}$for all the samples studied were calculated. The highest values of $n, E$ and $A$ were obtained for the composites filled with WRHA and AR. A linear dependence between $\ln A$ and $E$ was observed, known also as kinetic compensation effect. The results obtained were considered enough to conclude that the cheap RRH and the products of its thermal degradation BRHA and WRHA, after vigorously grounding and mixing, could successfully be used as fillers for polypropene instead of the much more expensive synthetic material Aerosil to prepare various polypropene composites.
\end{abstract}

Keywords: polymer composites, rice husks, thermal properties, non-isothermal degradation, kinetics

\section{Introduction}

Polypropene (PP) is a major thermoplastic stereoregular polymer, widely used in many industries construction, automotive, computer components, wire and cable, home appliances, food packing, medical bags, etc. [1-5]. The versatility of polypropene is due to its good chemical resistance, low density and a relatively high melting point, so it is useful for storing hot liquids. This polymer represents nowadays about 22 mass $\%$ of the demand for thermoplastics in the world, with a strong increase tendency $[5,6]$.

The thermal stability, thermal and oxidative degradation of virgin and filled polypropene composites is important from different points of view. On one hand, in a number of cases, it is necessary to increase its heat resistance by introducing certain reinforcing additives. On another hand, introduced reinforcing additives lead to an increase of the tensile strength of the composite with respect to the polymer matrix, but depending on the fillers nature the thermal stability of the composites may increase or decrease.

Incorporation of rigid inorganic particles is a promising approach to improve both stiffness and toughness of plastics simultaneously [3]. For example, polypropene composites used for insulation of electrical power distribution and transmission cables operating at temperatures higher than $70^{\circ} \mathrm{C}$ and under high electric field must be heat resistant, because the excess of the temperature rise in the cable is one of the main causes of dielectric failure [7]. On the other hand, polypropene constitutes are the main components of plastic waste from indus-

*Corresponding author, e-mail: sturmanova@btu.bg

(C) BME-PT and GTE 
trial and domestic refuse. In order to recycle plastic waste to obtain energy or low molecular hydrocarbons, other additives, for example solids, contained Lewis and Brǿnsted acid centers, are used to decrease the thermal stability of the composites [8-10]. Therefore, comprehension of the kinetics of its thermal degradation is of great interest for both theory and practice.

Polypropene is usually used in industry in the form of composites containing ingredients different by nature and in quantity, which improve its thermal and physico-mechanical properties, rather than in pure form. The addition of fillers to the polymer matrix is a fast and cheap method to modify the properties of the base polymer. Among the mineral fillers for PP calcium carbonate, talc, mica, silica, zinc oxide, glass, carbon black and carbon fibers are the most often used $[10,11]$. Each filler adds its own characteristics to the matrix and, as a consequence, changes the properties of the composite. In the recent years, natural fibers and flours have been widely used as reinforcing fillers in thermoplastic polymer composite materials $[12,13]$. These composites have several advantages, such as low cost, renewability, biodegradability and absence of associated health hazards. Agricultural residues such as rice husks; bagasse and wood chips are particularly important natural resources. Rice husks are an agricultural industrial residue produced as by-products during the rice-milling process. According to FAO statistical data [13], the annual world rice production was approximately 600 million tons in 2000, $20 \%$ of which was wasted as rice husks. Raw rice husks contain cellulose $35 \%$, hemicellulose $25 \%$, lignin $20 \%$, ash $17 \%$ (94\% silica) and moisture about $3 \%$ by weight [14]. Most of the rice husks are either used as a bedding material for animals and burned or used for landfilling. Therefore, the use of rice husks and its derivates in the manufacturing of thermoplastic polymer composites is attracting much attention. The rice husks ash, which is produced by burning of raw rice husks, has a high content of silica and may be used as a filler of different thermoplastics. Depending on the procedure employed during burning, two types of ashes are produced, i.e. white (WRHA) and black rice husks ash (BRHA). Much work [14-16] has been done to study the potential of raw rice husks flour RRHF, WRHA or BRHA as a filler of polyethylene or polypropene composites.
The disposal of waste polymers and particularly polypropene is a major environmental problem. Because they are not easily biologically degradable and because of their weight-to-volume ratios which creates problems of disposal, the plastics are not appropriate candidates for landfilling [8, 9, 12, 17]. Nowadays, incineration is the more widely used way to eliminate solid residues and it is also used for generation of electric and calorific energy at the same time. Uncontrolled incineration, however, can produce serious air pollution, environmental and health problems due to the possible emission of toxic flue gases (dioxins, furans), acid gases and heavy metals $[5,6,18]$. For this reason, the search for different recycling alternatives (thermal or oxidative degradation) is more and more necessary $[19,20]$. One of the possible ways to treat this waste is thermal decomposition - a process yielding both energy and gaseous and liquid products, which can be further used in various ways. A recently reported alternative is to transform the polymer waste into hydrocarbons in order to produce low molecular mass chemicals, clean hydrocarbon, chemical resources, fuels, gasoline, or other valuable products such as lubricants by thermal or catalytic degradation. The thermal degradation (pyrolysis) of the polymer macromolecules in the absence of oxygen or under vacuum at elevated temperatures gives a mixture of hydrocarbons $[5,6$, $8,12,18,21]$. According to the data from the gas chromatography/mass spectrometry analyses (GC/ MS), the thermal degradation of polypropene in nitrogen medium gives dienes, alkanes, and alkenes up to $\mathrm{C}_{31}$ hydrocarbons [17]. However, this degradation requires a considerable quantity of energy due to its endothermic character and the low thermal conductivities of the plastic materials throughout the process. Alternatively, some solid catalysts have been proposed to decrease degradation temperature and reduce this energy requirement [22, 23]. On the other hand, solid catalysts used in the process of thermal degradation of plastics increase the yield of liquid products. Catalytic degradation requires lower temperatures, and the chemical distribution of the product is narrower than in thermal degradation, leading to the production of more valuable products. Typical catalysts used for polymer degradation are acidic solids like amorphous alumina, silica-aluminas, clays, synthetic or natural zeolites (clinoptilolite, montmorillonite, atta- 
pulgite), mesoporous materials and activated carbon [3, 5, 6,1 8, 23-25]. Their behaviors may be due to the presence of Brónsted and Lewis strong acid sites, which can promote polymer chain cracking. In all these cases, the use of a catalyst reduces the energetic demand of the process and improves, from the economic point of view, the product distribution by increasing the yield of more volatile products and lowering the yield of subproducts. An important feature of catalytic degradation is the need for proper contact between the polymer and the catalyst to minimize degradation residues. However, all these catalysts are expensive and, at the moment, there are no useful ways to improve their short life and difficult recycling, so their use involves increased cost of the operational process. Thermogravimetric analysis (TGA) is one of the thermal analysis techniques used to measure the mass change, thermal decomposition and thermal stability of composite materials. Overall kinetics can be easily obtained by measuring the change in mass of a sample with time based on isothermal or non-isothermal thermogravimetric data $[2,4,8,19$, 26-29]. Some valuable parameters, such as apparent activation energy $E$, pre-exponential factor $A$, and reaction order $n$ can be calculated from a thermogravimetric curve. Knowledge of the kinetic parameters associated with thermal degradation constitutes and important tool in estimating the thermal behavior of composites under dynamic conditions.

The purpose of this study is to evaluate the thermal stability of polypropene composites filled with 20 mass\% raw rice husks, black or white rice husks ash, as well as establish the most appropriate mechanism of the thermal degradation and calculate the kinetic parameters characterizing this process.

\section{Experimental part}

\subsection{Materials and measurements}

The thermoplastic polymer polypropene (PP) 'Buplen' grade 6531, commercial product of Lukoil-Nephtochim (Bourgas, Bulgaria) with melt index $3-5 \mathrm{~g} / 10 \mathrm{~min}\left(230^{\circ} \mathrm{C} / 2160 \mathrm{~g}\right)$ was used as matrix for the preparation of the composites. The fillers used were raw rice husks (RRH), black rice husks ash (BRHA), white rice husks ash (WRHA) and, for comparison, Aerosil A200 (AR), product of Degussa AG (Germany).
Rice husks were obtained from suburb areas of Thrace (Pazardjik, Bulgaria). Before use, the rice husks were thoroughly washed - two times with tap water followed by three times with deionised water to remove adhering soil, clay and dust and finally dried at $102 \pm 2{ }^{\circ} \mathrm{C}$ overnight. The dried husks were ground in rotary cutting mill and sieved manually with $0.63-0.12 \mathrm{~mm}$ sieves. This starting material was used for thermal decomposition in air or nitrogen atmosphere. The thermal treatment of the rice husk fraction was carried out in quartz vertical tube, equipped with perforated quartz diaphragm at its lower end. The electrical heater used was wound around the quartz tube and insulated by asbestos sleeve. During the thermal treatment, nitrogen (or air) was flown below the quartz diaphragm into the lower end of the tube $\left(100 \mathrm{~cm}^{3} \cdot \mathrm{min}^{-1}\right)$ through the treated material. In the case of thermal treatment of raw rice husks in nitrogen medium, black rice husks ash (BRHA) was obtained and in air atmosphere - white rice husks ash (WRHA), respectively. The controlled increase of the temperature was performed using electronic thermal regulator Zeitplansollwertgeber, (Germany) at heating rate of $2^{\circ} \mathrm{C} \cdot \mathrm{min}^{-1}$ up to $700^{\circ} \mathrm{C}$, maintained for 2 hours, after which the ash was quenched in nitrogen or air medium and used for further experiments. All the samples were stored in plastic sealer containers and used after vigorous grinding.

Polypropene composites with 20 mass\% different fillers (RRH, BRHA, WRHA and AR) were prepared. The polymer and the filler were mixed with laboratory mixing rolls at $170^{\circ} \mathrm{C}$ for $5 \mathrm{~min}$. The compositions obtained were pressed on an electrically heated hydraulic laboratory press PHI (England) as a slab with thickness of $1 \mathrm{~mm}$. The slab was first cut into chips less than $1 \mathrm{~mm}$ long and then used for the thermogravimetric measurements. These samples were studied in a dynamic flow of air at a rate of $30 \mathrm{~cm}^{3} \cdot \mathrm{min}^{-1}$ under non-isothermal conditions in a derivatograph system PaulikPaulik-Erdey (MOM, Hungary) at heating rate of $10 \mathrm{~K} \cdot \mathrm{min}^{-1}$ in the temperature region $25-600^{\circ} \mathrm{C}$. Samples of $50 \mathrm{mg}$ were weighted into a alumina crucible and put in it without pressing. $\alpha$-Alumina calcined up to $1100^{\circ} \mathrm{C}$ was used as a standard reference material. The TG, DTA and DTG curves were recorded graphically with $1 \mathrm{mg}$ sensitivity. The test was performed at least twice for each sample to ensure its reproducibility. 


\subsection{Theoretical approach and calculation procedures}

The kinetics of thermal degradation reactions is described by various equations taking into account the special features of their mechanisms. The reaction rate can be expressed through the degree of conversion $\alpha$ according to the Equation (1):

$\alpha=\frac{m_{0}-m_{i}}{m_{0}-m_{f}}$

where $m_{0}, m_{f}$ and $m_{i}$ are initial, final and current sample mass at the moment $t$, respectively. Generally, the kinetic equation of the process can be written as Equation (2) [2, 27-30]:

$\frac{\mathrm{d} \alpha}{\mathrm{d} t}=k(T) f(\alpha)$

where $f(\alpha)$ is the conversion function and $k(T)$ is the temperature function respectively. The temperature dependence of the rate constant $k$ for the process is described by the Arrhenius equation (3):

$k=A \exp \left(-\frac{E}{R T}\right)$

where $A$ is the pre-exponential factor, $T$ is the absolute temperature, $R$ is the universal gas constant, and $E$ is the apparent activation energy of the process. Substitution of Equation (3) in (2) gives the Equation (4):

$$
\frac{\mathrm{d} \alpha}{\mathrm{d} t}=A \exp \left(-\frac{E}{R T}\right) f(\alpha)
$$

When the temperature increases at a constant rate (Equation (5)):

$\frac{\mathrm{d} T}{\mathrm{~d} t}=q=$ constant

therefore Equation (6):

$\frac{\mathrm{d} \alpha}{\mathrm{d} T}=\frac{A}{q} \exp \left(-\frac{E}{R T}\right) f(\alpha)$

The conversion function $f(\alpha)$ for a solid-state reaction depends on the reaction mechanism and can generally be considered to be as Equation (7):

$$
f(\alpha)=\alpha^{m}(1-\alpha)^{n}[-\ln (1-\alpha)]^{p}
$$

where $m, n$ and $p$ are empirically obtained exponent factors, one of them always being zero [30, 31].
After substitution in Equation (6), separation of variables and integration, the following general equation (8) was obtained:

$$
\int_{0}^{\alpha} \frac{\mathrm{d} \alpha}{\alpha^{\mathrm{m}}(1-\alpha)^{\mathrm{n}}[-\ln (1-\alpha)]^{\mathrm{p}}}=\frac{A}{q} \int_{0}^{T} \exp \left(-\frac{E}{R T}\right) \mathrm{d} T
$$

The solutions of the left hand side integral depend on the explicit expression of the function $f(\alpha)$ and are denoted as $g(\alpha)$. Algebraic expressions of functions of the most common reaction mechanisms operating in solid-state reactions are summarized and presented in Table 1 [2, 4, 26, 30].

The right hand side integral in Equation (8) has no exact analytical solution and, after making some variable substitution, is expressed by the fourth Senum and Yang approximation (Equations (9) and (10)) $[32,33]$, which gives an accuracy better than $10^{-5 \%}$ for $x=E / R T \geq 20$.

$$
\begin{aligned}
& \frac{A}{q} \int_{0}^{T} \exp \left(-\frac{E}{R T}\right) \mathrm{d} T=\frac{A E}{q R} \frac{\exp (-x)}{x^{2}} h(x) \\
& h(x)=\frac{x^{4}+18 x^{3}+86 x^{2}+86 x}{x^{4}+20 x^{3}+120 x^{2}+240 x+120}
\end{aligned}
$$

The approximate expression was therefore used in the discussion of error of the activation energy estimated by the Coats and Redfern calculation procedure [34].

For Equation (4), considering the maximum reaction rate, leads to Equation (11):

$$
-\frac{1}{\frac{\mathrm{d} f(\alpha)}{\mathrm{d} \alpha}}=-\frac{1}{f^{\prime}\left(\alpha_{\max }\right)}=\frac{A}{q} \frac{R T_{\max }^{2}}{E} \exp \left(-\frac{E}{R T_{\max }}\right)
$$

The subscript 'max' denotes the variables at the maximum reaction rate. Furthermore, combining Equation (8) with Equation (11) gives the Equation (12):

$g\left(\alpha_{\max }\right) \cdot f^{\prime}\left(\alpha_{\max }\right)=-h\left(\frac{E}{R T_{\max }}\right)$

Equation (12) indicates that $\alpha_{\max }$ value depends only on $E / R T_{\max }$ value for a definite kinetic model. This quantitative relation can be applied to estimate the limits of $\alpha_{\max }$ for various kinetic models and 
Table 1. Algebraic expressions of functions $f(\alpha)$ and $g(\alpha)$ and its corresponding mechanism $[2,4,26,30]$

\begin{tabular}{|c|c|c|c|c|c|}
\hline No. & $\begin{array}{l}\text { Mecha- } \\
\text { nism }\end{array}$ & Name of the function & $\mathbf{g}(\alpha)$ & $f(\alpha)$ & $\begin{array}{l}\text { Rate-determining } \\
\text { mechanism }\end{array}$ \\
\hline \multicolumn{6}{|c|}{ 1. Chemical process or mechanism non-invoking equations } \\
\hline 1. & $\mathrm{~F}_{1 / 3}$ & One-third order & $1-(1-\alpha)^{2 / 3}$ & $(3 / 2)(1-\alpha)^{1 / 3}$ & Chemical reaction \\
\hline 2. & $\mathrm{~F}_{3 / 4}$ & Three-quarters order & $1-(1-\alpha)^{1 / 4}$ & $4(1-\alpha)^{3 / 4}$ & Chemical reaction \\
\hline 3. & $\mathrm{~F}_{3 / 2}$ & One and a half order & $(1-\alpha)^{-1 / 2}-1$ & $2(1-\alpha)^{3 / 2}$ & Chemical reaction \\
\hline 4. & $\mathrm{~F}_{2}$ & Second order & $(1-\alpha)^{-1}-1$ & $(1-\alpha)^{2}$ & Chemical reaction \\
\hline 5. & $\mathrm{~F}_{3}$ & Third order & $(1-\alpha)^{-2}-1$ & $(1 / 2)(1-\alpha)^{3}$ & Chemical reaction \\
\hline \multicolumn{6}{|c|}{ 2. Acceleratory rate equations } \\
\hline 6. & $\mathrm{P}_{3 / 2}$ & Mampel power law & $\alpha^{3 / 2}$ & $(2 / 3) \alpha^{-1 / 2}$ & Nucleation \\
\hline 7. & $\mathrm{P}_{1 / 2}$ & Mampel power law & $\alpha^{1 / 2}$ & $2 \alpha^{1 / 2}$ & Nucleation \\
\hline 8. & $\mathrm{P}_{1 / 3}$ & Mampel power law & $\alpha^{1 / 3}$ & $3 \alpha^{2 / 3}$ & Nucleation \\
\hline 9. & $\mathrm{P}_{1 / 4}$ & Mampel power law & $\alpha^{1 / 4}$ & $4 \alpha^{3 / 4}$ & Nucleation \\
\hline 10. & $\mathrm{E}_{1}$ & Exponential law & $\ln \alpha$ & $\alpha$ & Nucleation \\
\hline \multicolumn{6}{|c|}{ 3. Sigmoidl rate equations or random nucleation and subsequent growth } \\
\hline 11. & $\mathrm{~A}_{1}, \mathrm{~F}_{1}$ & Avrami-Erofeev eq. & $-\ln (1-\alpha)$ & $1-\alpha$ & $\begin{array}{l}\text { Assumed random nucleation } \\
\text { and its subsequent growth, } \\
n=1\end{array}$ \\
\hline 12. & $\mathrm{~A}_{3 / 2}$ & Avrami-Erofeev eq. & {$[-\ln (1-\alpha)]^{2 / 3}$} & $(3 / 2)(1-\alpha)[-\ln (1-\alpha)]^{1 / 3}$ & $\begin{array}{l}\text { Assumed random nucleation } \\
\text { and its subsequent growth, } \\
n=1.5\end{array}$ \\
\hline 13. & $\mathrm{~A}_{2}$ & Avrami-Erofeev eq. & {$[-\ln (1-\alpha)]^{1 / 2}$} & $2(1-\alpha)[-\ln (1-\alpha)]^{1 / 2}$ & $\begin{array}{l}\text { Assumed random nucleation } \\
\text { and its subsequent growth, } \\
n=2\end{array}$ \\
\hline 14. & $\mathrm{~A}_{3}$ & Avrami-Erofeev eq. & {$[-\ln (1-\alpha)]^{1 / 3}$} & $3(1-\alpha)[-\ln (1-\alpha)]^{2 / 3}$ & $\begin{array}{l}\text { Assumed random nucleation } \\
\text { and its subsequent growth, } \\
n=3\end{array}$ \\
\hline 15. & $\mathrm{~A}_{4}$ & Avrami-Erofeev eq. & {$[-\ln (1-\alpha)]^{1 / 4}$} & $4(1-\alpha)[-\ln (1-\alpha)]^{3 / 4}$ & $\begin{array}{l}\text { Assumed random nucleation } \\
\text { and its subsequent growth, } \\
n=4\end{array}$ \\
\hline 16. & $\mathrm{~A}_{\mathrm{u}}$ & Prout-Tomkins eq. & $\ln [\alpha /(1-\alpha)]$ & $\alpha(1-\alpha)$ & Branching nuclei \\
\hline \multicolumn{6}{|c|}{$\begin{array}{l}\text { 4. Deceleratory rate equations } \\
\text { 4.1. Phase boundary reaction }\end{array}$} \\
\hline 17. & $\mathrm{R}_{1}, \mathrm{~F}_{0}, \mathrm{P}_{1}$ & Power law & $\alpha$ & $(1-\alpha)^{0}$ & Contracting disk \\
\hline 18. & $\mathrm{R}_{2}, \mathrm{~F}_{1 / 2}$ & Power law & $1-(1-\alpha)^{1 / 2}$ & $2(1-\alpha)^{1 / 2}$ & $\begin{array}{l}\text { Contracting cylinder } \\
\text { (cylindrical symmetry) }\end{array}$ \\
\hline 19. & $\mathrm{R}_{3}, \mathrm{~F}_{2 / 3}$ & Power law & $1-(1-\alpha)^{1 / 3}$ & $3(1-\alpha)^{2 / 3}$ & $\begin{array}{l}\text { Contracting sphere } \\
\text { (spherical symmetry) }\end{array}$ \\
\hline \multicolumn{6}{|c|}{ 4.2. Based on the diffusion mechanism } \\
\hline 20. & $\mathrm{D}_{1}$ & Parabola low & $\alpha^{2}$ & $1 / 2 \alpha$ & One-dimensional diffusion \\
\hline 21. & $\mathrm{D}_{2}$ & Valensi eq. & $\alpha+(1-\alpha) \ln (1-\alpha)$ & {$[-\ln (1-\alpha)]^{-1}$} & Two-dimensional diffusion \\
\hline 22. & $D_{3}$ & Jander eq. & {$\left[1-(1-\alpha)^{1 / 3}\right]^{2}$} & $(3 / 2)(1-\alpha)^{2 / 3}\left[1-(1-\alpha)^{1 / 3}\right]^{-1}$ & $\begin{array}{l}\text { Three-dimensional diffusion, } \\
\text { spherical symmetry }\end{array}$ \\
\hline 23. & $\mathrm{D}_{4}$ & Ginstling-Brounstein eq. & $1-2 \alpha / 3-(1-\alpha)^{2 / 3}$ & $(3 / 2)\left[(1-\alpha)^{-1 / 3}-1\right]^{-1}$ & $\begin{array}{l}\text { Three-dimensional diffusion, } \\
\text { cylindrical symmetry }\end{array}$ \\
\hline 24. & $D_{5}$ & $\begin{array}{l}\text { Zhuravlev, Lesokin, } \\
\text { Tempelman eq. }\end{array}$ & {$\left[(1-\alpha)^{-1 / 3}-1\right]^{2}$} & $(3 / 2)(1-\alpha)^{4 / 3}\left[(1-\alpha)^{-1 / 3}-1\right]^{-1}$ & Three-dimensional diffusion \\
\hline 25. & $\mathrm{D}_{6}$ & anti-Jander eq. & {$\left[(1+\alpha)^{1 / 3}-1\right]^{2}$} & $(3 / 2)(1+\alpha)^{2 / 3}\left[(1+\alpha)^{1 / 3}-1\right]^{-1}$ & Three-dimensional diffusion \\
\hline 26. & & $\begin{array}{l}\text { anti-Ginstling-Brounstein } \\
\text { eq. }\end{array}$ & $1+2 \alpha / 3-(1+\alpha)^{2 / 3}$ & $(3 / 2)\left[(1+\alpha)^{-1 / 3}-1\right]^{-1}$ & Three-dimensional diffusion \\
\hline 27. & & $\begin{array}{l}\text { anti-Zhuravlev, Lesokin, } \\
\text { Tempelman eq. }\end{array}$ & {$\left[(1+\alpha)^{-1 / 3}-1\right]^{2}$} & $(3 / 2)(1+)^{4 / 3}\left[(1+\alpha)^{-1 / 3}-1\right]^{-1}$ & Three-dimensional diffusion \\
\hline
\end{tabular}

then classify the kinetic models by the magnitude of $\alpha_{\max }$. This is because ordinary reactions have $E / R T=15-70$, correspondingly $h(E / R T)=0.8879$
0.9726. For $n$-th order reactions $f(\alpha)=(1-\alpha)^{n}$, Equation (12) thus turns into Equations (13) and (14). 
$1-\alpha_{\max }=\left[1-\frac{n-1}{n} h\left(\frac{E}{R T_{\max }}\right)\right]^{1 /(n-1)} \quad$ for $\quad n \neq 1$

and

$-\ln \left(1-\alpha_{\max }\right)=h\left(\frac{E}{R T_{\max }}\right) \quad$ for $n=1$

Based on Equations (13) and (14), the limits of $\alpha_{\max }$ at various $n$ values are estimated and listed in Table 2, which is useful to determine the reaction order from experimental $\alpha_{\max }$ value [32, 33].

For the present study, the calculation procedure of Coats and Redfern [34] was used. Data from TG and DTG curves in the decomposition range 0.10.9 were used to determinate the kinetic parameters of the process. The kinetic parameters can be derived using a modified Coats and Redfern equation (15):

$\ln \frac{g(\alpha)}{T^{2}}=\ln \frac{A R}{q E}-\frac{E}{R T}$

where $g(\alpha)$ is a function, the expression of which depends on the kinetic model of the occurring reaction. If the correct $g(\alpha)$ is used, a plot of $\ln \left[g(\alpha) / T^{2}\right]$ against $1 / T$ should give a straight line from which the values of the activation energy $E$ and the preexponential factor $A$ in Arrhenius equation can be calculated. The formal expressions of the functions $g(\alpha)$ depend on the conversion mechanism and its mathematical model [2, 27-30]. The latter usually represents the limiting stage of the reaction - the chemical reactions; random nucleation and nuclei growth; phase boundary reaction or diffusion. For the correct $g(\alpha)$ (Table 1), the corresponding linear

Table 2. The theoretical limits* of $\alpha_{\max }$ of $n$-th order reactions at various $n$ values

\begin{tabular}{|c|c|c|c|c|}
\hline $\boldsymbol{\alpha}_{\max }$ & $\mathbf{n}$ & $\boldsymbol{\alpha}_{\max }$ & $\mathbf{n}$ \\
\hline $0.913-0.920$ & 0.1 & & $0.569-0.604$ & 1.1 \\
\hline $0.850-0.862$ & 0.2 & $0.551-0.587$ & 1.2 \\
\hline $0.799-0.816$ & 0.3 & $0.534-0.571$ & 1.3 \\
\hline $0.756-0.777$ & 0.4 & $0.519-0.557$ & 1.4 \\
\hline $0.719-0.743$ & 0.5 & $0.504-0.543$ & 1.5 \\
\hline $0.687-0.713$ & 0.6 & $0.491-0.531$ & 1.6 \\
\hline $0.659-0.687$ & 0.7 & $0.478-0.519$ & 1.7 \\
\hline $0.633-0.663$ & 0.8 & $0.466-0.507$ & 1.8 \\
\hline $0.610-0.642$ & 0.9 & $0.455-0.496$ & 1.9 \\
\hline $0.588-0.622$ & 1.0 & $0.444-0.486$ & 2.0 \\
\hline
\end{tabular}

*Corresponding to $E / R T=15 \div 70$ dependence should give the highest correlation coefficient at the linear regression analysis.

Later, a number of authors [35-36] suggested different solutions for the temperature integral in Equation (8), insisting that they increase the accuracy with which the kinetic parameters are calculated. For instance, Madhysudanan - Krishnan Ninan [35] suggested the Equation (16):

$$
\begin{aligned}
& \ln \left[\frac{g(\alpha)}{T^{1.921503}}\right]= \\
& {\left[\ln \frac{A R}{q R}+3.772050-1.921503 \ln E\right]-0.120394 \frac{E}{T}}
\end{aligned}
$$

Tang et al. [36] offered another kinetic equation (17):

$$
\begin{aligned}
\ln \left[\frac{g(\alpha)}{T^{1.894661}}\right]= \\
=\left[\ln \frac{A R}{q R}+3.63504095-1.894661 \ln E\right]- \\
\quad 0.00145033 \frac{E}{T}
\end{aligned}
$$

and Wanjun et al. [37] used the Equation (18):

$$
\begin{aligned}
& \ln \left[\frac{g(\alpha)}{T^{2}}\right]= \\
& =\left[\ln \frac{A E}{q\left(1.00198882 E+1.87391198 R T_{p}\right)}\right]-\frac{E}{R T}
\end{aligned}
$$

Equations (15-18) suggest certain differences between the calculated values of the apparent activation energy $E$ and pre-exponential factor $A$ even when the same $g(\alpha)$ function is used. To estimate which of these four calculation procedures would be most adequate for the computations, the maximum value of the linear regression correlation coefficient $R^{2}$ was used. Concerning the calculations of the kinetic parameters, a computer program was developed for all the data manipulations.

The values of the pre-exponential factor $A$ in Arrhenius equation for solid phase reactions are expected to be in wide range (six or seven orders of magnitude), even after the effect of surface area is corrected for $[28,30]$. Empirical first order pre-expo- 
nential factors may vary from $10^{4}$ to $10^{18} \mathrm{~s}^{-1}$. The low factors will often indicate a surface reaction, but if the reactions are not dependent on surface area, the low factor may indicate a 'tight' complex. The high factors will usually indicate a 'loose' complex. Even higher factors (after correction of surface area) can be obtained if the complexes have free translation on the surface. Since the concentrations in the solid are not controllable in many cases, it would have been convenient if the magnitude of the pre-exponential gave an indication of the molecularity. This appears to be true only for nonsurface-controlled reactions having low $\left(<10^{9} \mathrm{~s}^{-1}\right)$ preexponential factors. Such reactions (if elementary) can only be bimolecular.

From the theory of the activated complex (transition state) of Eyring [27-30], it is known that (Equation (19)):

$A=\frac{e \chi k_{B} T_{p}}{h} \exp \left(\frac{\Delta S^{\neq}}{R}\right)$

where $\mathrm{e}=2.7183$ is the Neper number; $\chi$ - transmission factor, which is unity for monomolecular reactions; $k_{B}$ - Boltzmann constant; $h$ - Plank constant, and $T_{p}$ is the peak temperature of the DTA curve. The change of entropy $\Delta S^{ \pm}$may be calculated according to the Equations (20) and (21) [28, 30]:

$\Delta S^{\neq}=R \ln \frac{A h}{e \chi k_{B} T_{p}}$

Since

$\Delta H^{\neq}=E-R T_{p}$

the changes of the enthalpy $\Delta H^{\neq}$and Gibbs free energy $\Delta G^{\neq}$for the activated complex formation from the reagent can be calculated using the well known thermodynamic equation (22):

$\Delta G^{\neq}=\Delta H^{\neq}-T_{p} \Delta S^{\neq}$

When studying the kinetics of reactions of the same type using the correct $g(\alpha)$ function, the straight lines characterizing the kinetics of these reactions usually cross at a point with co-ordinates $\ln k_{i s o}$ and $1 / T_{\text {iso. }}$. This point corresponds to the so-called isokinetic temperature $T_{i s o}$, at which the rate constants of the reactions have the same value $-k_{i s o}$. This means that at temperatures higher than $T_{i s o}$, the reactions occurring at higher $E$ would have higher rate (higher rate constants) than these with lower activation energies. It turned out that the following linear dependence exists between $\ln A$ and $E$ in these cases (Equation (23)):

$\ln A=\ln k_{i s o}+\frac{E}{R T_{i s o}}$

which is known also as kinetic compensation effect, isokinetic effect, or $\theta$ - rule [28, 38, 39-41]. Besides, linear dependence between $E$ and $\Delta S^{\ddagger}$ can also be observed [38].

\section{Results and discussion}

The controlled burning of the raw rice husks in air atmosphere lead to production of white rice husk ash (WRHA) or so-called 'white ash', which contained almost pure $(\geq 95 \%)$ silica in a hydrated amorphous form, similar to silica gel, with high porosity and reactivity and about 5 mass $\%$ inorganic impurities as an aluminium, calcium, magnesium and iron oxides. The controlled pyrolysis of the raw rice husks in nitrogen medium leads to production of black rice husk ash (BRHA) or so-called 'black ash', which contained 45 mass\% carbon, 50 mass $\%$ silica and about 5 mass $\%$ inorganic impurities. According to the data from X-ray analysis, all the fillers used were amorphous. The physicochemical characteristics of the fillers used are shown in Table 3.

Table 3. Physicochemical characteristics of the RRH, WRHA, BRHA and AR

\begin{tabular}{|l|c|c|c|c|}
\hline \multicolumn{1}{|c|}{ Parameter } & RRH & WRHA & BRHA & AR \\
\hline $\mathrm{SiO}_{2}[$ mass\%] & 20.2 & 94.2 & 54.0 & 100 \\
\hline Moisture $[\mathrm{mass} \%]$ & 7.1 & $<0.5$ & $<0.5$ & $<0.5$ \\
\hline Mean particle size $[\mu \mathrm{m}]$ & 80 & 50 & 60 & 20 \\
\hline Surface area $\left[\mathrm{m}^{2} \cdot \mathrm{g}^{-1}\right]$ & $<1$ & 228 & 141 & 273 \\
\hline True density $\left[\mathrm{g} \cdot \mathrm{cm}^{-3}\right]$ & 1.47 & 2.2 & 1.8 & 2.2 \\
\hline
\end{tabular}

\subsection{Kinetic studies}

The thermogravimetric curves of pure polypropene and polypropene filled with 20 mass $\%$ different by nature fillers measured at heating rate of $10 \mathrm{~K} \cdot \mathrm{min}^{-1}$ are presented in Figure 1. As it can be seen from the figure, the shapes of the TG curves are quite different. As far as the polymer is concerned, its degrada- 


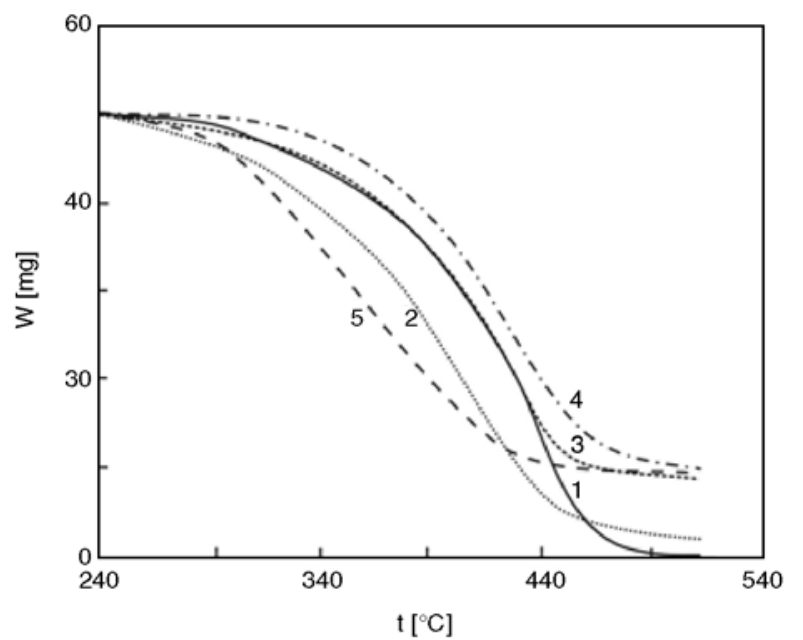

Figure 1. TG curves of pure polypropene - 1, polypropene filled with 20 mass $\%$ RRH - 2, BRHA - 3, WRHA - 4 and AR -5

tion process obeys generally the sigmoidal or deceleration functions [2]. Pure polypropene decomposes without formation of solid residue (curve 1) while the other samples produce various amounts of solid residue of amorphous $\mathrm{SiO}_{2}$ depending on the nature of the filler used (curves 2-5). Besides, the TG curves are shifted to different extents vs. that of the pure polypropene. For example, the TGcurve for polypropene filled with AR (curve 5) is shifted toward lower temperatures and the thermal degradation is complete at temperatures up to $440^{\circ} \mathrm{C}$, obtaining solid residue of about 20 mass $\%$. It may be concluded; therefore, that Aerosil as a filler of polypropene decreases its thermal stability most probably due to the monolayer distribution of PP on the filler and the presence of certain acidic centers on its surface [23]. The TG curve of polypropene filled with RRH (curve 2) is shifted towards lower temperature $v s$. that of the pure polypropene because the organic part of the raw rice husks started to decompose at temperature lower than that of pure polypropene [27]. The TG curve of polypropene filled with BRHA (curve 3 ) is practically the same as that for pure polypropene (curve 1). It was attributed to the carbon coating formed on the surface of the amorphous $\mathrm{SiO}_{2}$, which makes it more compatible with the polymer matrix. For the characterization of polypropene composites thermal stability, two divergences among the studies are obvious: the first is the choice of kinetic models used to describe the pyrolysis kinetics, and the second is the calculation of

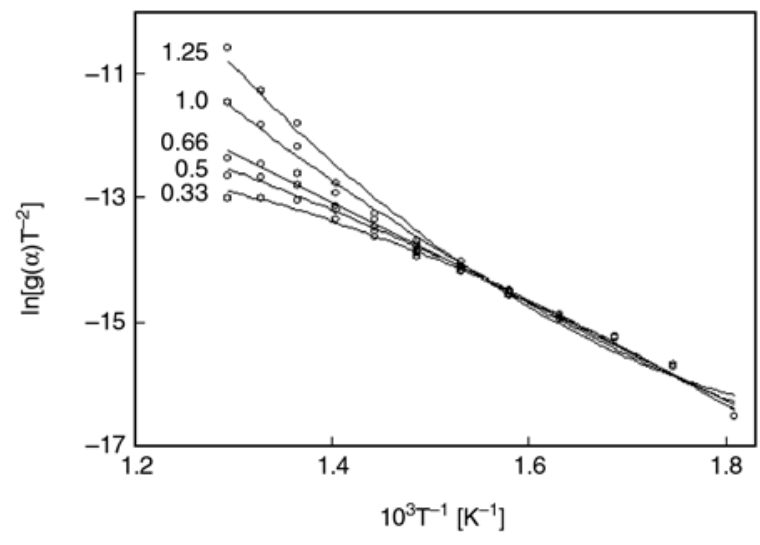

Figure 2. Dependence of $\ln \left[g(\alpha) / T^{2}\right]$ against $1 / T$ for pure polypropene at different values of $n$

the kinetic parameters obtained on the basis of the chosen model.

Using the calculation procedures of Coats and Redfern [34], all $g(\alpha)$ functions presented in Table 1 were first substituted into Equation (15) and the plot of the left side of this equation against $1 / T$ was fitted by computer to calculate the correlation coefficient of linear regression $R^{2}$. This procedure was repeated until the best $R^{2}$ value was obtained. For the thermal degradation of the samples studied, single heating rate plots, $\ln \left[g(\alpha) / T^{2}\right]$ against $1 / T$, were calculated according to Equation (15). It should be noted for all the samples studied that the highest values of $R^{2}$ were obtained when kinetic equations for the $F_{n}$ mechanism (Table 1 ) with different values of $n$ were used. In this respect, the kinetic curves of thermal degradation of pure polypropene with different values of $n$ are presented in Figure 2 for illustration.

Figure 2 shows that the shapes of the kinetic curves strongly depend on the value of the parameter $n$. The same is true also for the other four-polypropene composites. To find the value of $n$ with which the highest value of $R^{2}$ is obtained, the dependence $R^{2}=f(n)$ was drawn. For comparison, the corresponding curves for pure polypropene (curve 1) and polypropene composite filled with 20 mass\% Aerosil (curve 2) are presented in Figure 3.

The curves presented in Figure 3 can be described well with empiric polynomials of second order. Differentiating these polynomials vs. $n$ and assuming their value to be zero, the value of $n$ at which $R^{2}$ has maximum value could be calculated. For the first curve, the maximum of $R^{2}$ was obtained at $n=0.66$, 


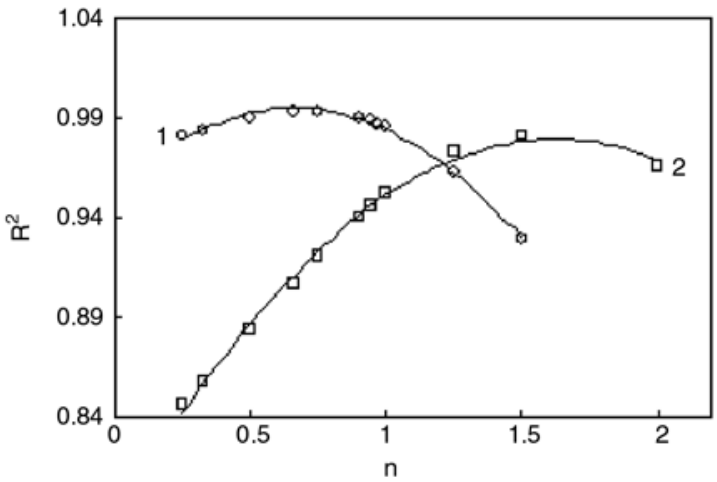

Figure 3. Dependence of the coefficient of linear regression $R^{2}$ on the values of $n$ for thermal degradation of: pure polypropene -1 and polypropene filled with 20 mass\% Aerosil - 2

while for the second curve at $n=1.63$. Similar procedure was used also to find the optimal value of $n$ for the other composites. Subsequently, the apparent activation energy $E$ and the pre-exponential factor $A$ can be calculated from the slope and intercept of the fitted straight line. The same method was used to process the data from the TG curves of the composites studied, using the calculation procedures of Madhysudanan et al. [35], Tang et al. [36] and Wanjun et al.[37]. The results obtained are summarized in Table 4.

As can be seen from Table 4, the value of $n$ varied from 0.66 to 1.63 , depending on filler nature. The higher values were observed for the samples filled with WRHA or AR, which are pure $\mathrm{SiO}_{2}$, by nature. The practically equal values of the kinetic parameters characterizing the thermal degradation of pure polypropene and polypropene filled with BRHA, as well as the same form of the kinetic equation $(n=0.66)$ confirmed the full compatibility of this filler with the matrix. In our opinion, the almost twice lower value of the activation energy of thermal destruction of the PP-BRHA sample compared to that of the PP-WRHA sample was due to the significant amount of porous carbon in BRHA. This porous carbon easily burns in oxidative medium (air) and facilitates the thermal degradation of the polypropene matrix. This is sustained by the lower activation energy of thermal destruction of PP-BRHA sample compared to pure polypropene, despite that the decomposition mechanism is the same $(n=0.66)$.

Since the values of $E$ and $A$ calculated using the four calculation procedures were quite close, they can be averaged. On this basis, the values of $\Delta S^{\neq}$, $\Delta H^{\neq}$and $\Delta G^{\neq}$were calculated at $T=T_{p}$, since this temperature characterizes the highest rate of the process, and therefore, is its important parameter. The change of Gibbs free energy $\Delta G^{\neq}$reflects the total energy increase of the system at the approach of the reagents and the formation of the activated complex. This energy is influenced by two thermodynamic properties, the changes of enthalpy $\Delta H^{\ddagger}$

Table 4. Kinetic parameters of non-isothermal degradation of filled with different fillers (20 mass \%) polypropene composites

\begin{tabular}{|c|c|c|c|c|c|}
\hline \multirow{2}{*}{ Parameter } & \multicolumn{5}{|c|}{ Sample } \\
\hline & $\mathbf{P P}$ & PP-RRH & PP-BRHA & PP-WRHA & PP-AR \\
\hline \multicolumn{6}{|c|}{ Coats - Redfern } \\
\hline$n$ & 0.66 & 1.05 & 0.66 & 1.28 & 1.63 \\
\hline$R^{2}$ & 0.9976 & 0.9968 & 0.9973 & 0.9982 & 0.9978 \\
\hline$E\left[\mathrm{~kJ} \cdot \mathrm{mol}^{-1}\right]$ & 66.7 & 71.3 & 63.3 & 108.0 & 112.6 \\
\hline$A\left[\mathrm{~min}^{-1}\right]$ & $1.26 \cdot 10^{4}$ & $6.78 \cdot 10^{4}$ & $7.99 \cdot 10^{3}$ & $3.95 \cdot 10^{7}$ & $7.20 \cdot 10^{8}$ \\
\hline \multicolumn{6}{|c|}{ Madhysudanan - Krishnan - Ninan } \\
\hline$E\left[\mathrm{~kJ} \cdot \mathrm{mol}^{-1}\right]$ & 66.7 & 71.5 & 63.6 & 109.7 & 112.7 \\
\hline$A\left[\mathrm{~min}^{-1}\right]$ & $1.5 \cdot 10^{4}$ & $8.08 \cdot 10^{4}$ & $9.81 \cdot 10^{3}$ & $5.57 \cdot 10^{7}$ & $8.28 \cdot 10^{8}$ \\
\hline \multicolumn{6}{|c|}{ Tang - Liu - Zang - Wang } \\
\hline$E\left[\mathrm{~kJ} \cdot \mathrm{mol}^{-1}\right]$ & 66.8 & 71.9 & 63.7 & 109.2 & 112.8 \\
\hline$A\left[\mathrm{~min}^{-1}\right]$ & $1.57 \cdot 10^{4}$ & $8.41 \cdot 10^{4}$ & $1.03 \cdot 10^{4}$ & $5.69 \cdot 10^{7}$ & $8.48 \cdot 10^{8}$ \\
\hline \multicolumn{6}{|c|}{ Wanjun - Yuwen - Hen - Zhiyong - Cunxin } \\
\hline$E\left[\mathrm{~kJ} \cdot \mathrm{mol}^{-1}\right]$ & 66.7 & 71.3 & 63.3 & 108.0 & 112.56 \\
\hline$A\left[\mathrm{~min}^{-1}\right]$ & $1.47 \cdot 10^{4}$ & $6.81 \cdot 10^{4}$ & $9.46 \cdot 10^{3}$ & $4.35 \cdot 10^{7}$ & $1.36 \cdot 10^{8}$ \\
\hline \multicolumn{6}{|c|}{ Average values } \\
\hline$E\left[\mathrm{~kJ} \cdot \mathrm{mol}^{-1}\right]$ & 66.7 & 71.5 & 63.5 & 108.7 & 112.7 \\
\hline$A\left[\mathrm{~min}^{-1}\right]$ & $1.45 \cdot 10^{4}$ & $7.52 \cdot 10^{4}$ & $9.39 \cdot 10^{3}$ & $4.89 \cdot 10^{7}$ & $6.33 \cdot 10^{8}$ \\
\hline$-\Delta S^{\neq}\left[\mathrm{J} \cdot \mathrm{mol}^{-1} \cdot \mathrm{K}^{-1}\right]$ & 214.9 & 200.9 & 218.4 & 147.3 & 125.1 \\
\hline$\Delta H^{\neq}\left[\mathrm{kJ} \cdot \mathrm{mol}^{-1}\right]$ & 60.7 & 65.8 & 57.6 & 102.8 & 107.4 \\
\hline$\Delta G^{\neq}\left[\mathrm{kJ} \cdot \mathrm{mol}^{-1}\right]$ & 216.1 & 205.0 & 212.2 & 207.8 & 187.2 \\
\hline
\end{tabular}


and entropy $\Delta S^{\ddagger}$ of activated complex formation. The change of activation enthalpy shows the energy differences between the activated complex and the reagents. If this difference is small, the formation of activated complex is favored, because the potential energy barrier is low. The change of reaction entropy reflects how near the system is to its own thermodynamic equilibrium. Low activation entropy means that the material has just passed through some kind of physical or chemical aging process, bringing it to a state near its own thermodynamic equilibrium. In this situation, the material shows little reactivity, increasing the time taken to form the activated complex. On the other hand, when high activation entropy values are observed, the material is far from its own thermodynamic equilibrium. In this case, the reactivity is high and the system can react faster to produce the activated complex, which resulted in the short reaction times observed [38].

The thermal degradation of pure polypropene has been described as occurring by a random scission mechanism or two-third-order kinetics model when the number of chain scission is measured to estimate the change in the degree of polymerization. In the cases of filled composites, however, when random scission degradation is measured by weight loss, the kinetics will probably deviate from a twothird-order model. Since not every broken bond leads to evaporation of products, only product fragments which are small enough to evaporate will actually evaporate and thus lead to a decrease in the sample mass. For this reason, deviation of kinetics of random scission degradation from a two-thirdorder reaction model may be observed for filled composites. Another reason for which the kinetic of the thermal degradation of filled composites may differ from the two-third order kinetics model $(n=$ $1.05)$ is that the degradation of PP in the samples filled with RRH was preceded by degradation of the organic component of the raw rice husks. For polypropene composites filled with WRHA or Aerosil, the kinetic model of decomposition is $F_{n}$, where $n=1.28$ and 1.63 respectively. This can be explained with the high specific area of the fillers and the possibility for adsorption of the polypropene decomposition products on their surface. The comparison of $R^{2}$ values obtained according to the different calculation procedures used showed that the results obtained by Coats - Redfern best

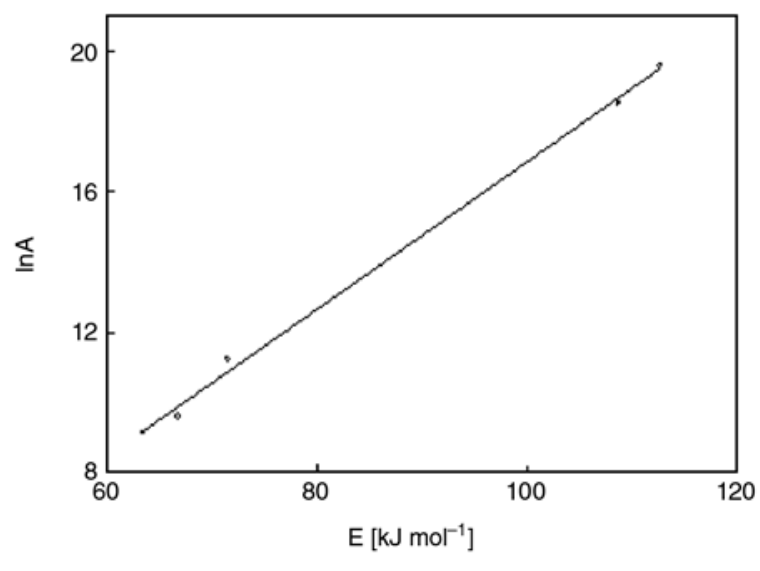

Figure 4. Compensation plot for the thermal decomposition of polypropene, filled with 20 mass $\%$ different fillers

describe the process of thermal decomposition reactions in pure and filled with different fillers polypropene.

On Figure 4 is presented the ccompensation plot for the thermal decomposition of polypropene, filled with 20 mass\% different fillers.

As can be seen in Figure 4, there is a linear dependence between $\ln A$ and $E$, which indicates for the existence of isokinetic relationship or kinetic compensation effect. It means that the most probable kinetic equations found describe correctly the mechanism of thermal destruction of the samples. The iso-kinetic rate constant $k_{\text {iso }}$ was calculated to be equal to $1.61 \cdot 10^{-2} \mathrm{~min}^{-1}$ and the iso-kinetic temperature $T_{\text {iso }}=574.4 \mathrm{~K}$. Above this temperature, the decomposition rate for the samples characterized by higher activation energy $E$ becomes higher than that of the samples decomposing at lower activation energy. Besides, linear dependence was

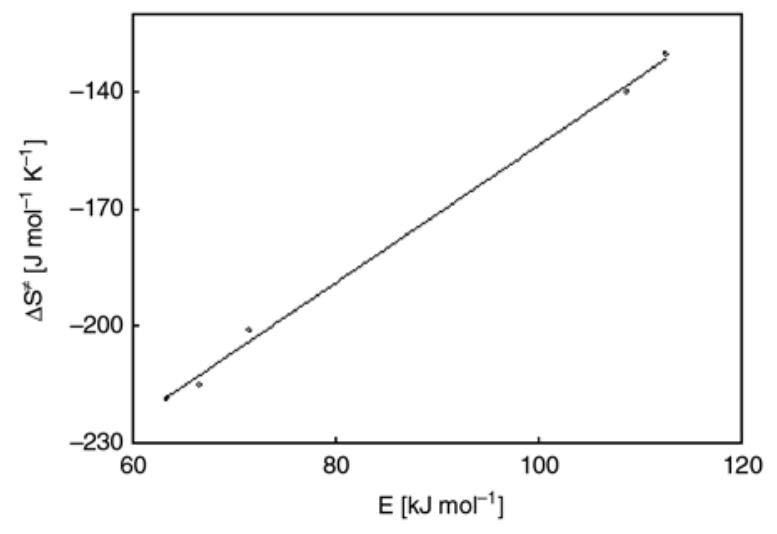

Figure 5. Plots of $\Delta S^{\ddagger} v s$. $E$ values for thermal decomposition of polypropene, filled with 20 mass $\%$ different fillers 
observed also between the change of the entropy for the formation of the activated complex $\Delta S^{\neq}$and the activation energy $E$ of the samples studied (Figure 5).

Figure 5 shows that the increase of the value of $E$ resulted in linear increase of the values of $\Delta S^{\neq}$, although remaining negative. It means that lower values of the entropy member correspond to higher values of the activation energy in the equation of Eyring, or the rearrangement of the initial structure of the composite during the formation of the activated complex was accompanied by smaller decrease of entropy. This effect was most evident for the samples filled with Aerosil and white rice husk ash, which are almost identical by chemical composition (pure $\mathrm{SiO}_{2}$ ) and so exert the same effect on the thermal stability of the filled composite materials.

\subsection{Mechanism of pyrolysis of polypropene}

A variety of reactions are possible in the thermal degradation of polypropene. According to some authors [42-46], the degradation may be described by the following reactions:

\section{Thermal degradation}

$\mathrm{P} \rightarrow \mathrm{R}_{r}^{\bullet}+\mathrm{R}_{n-r}^{\bullet}$

Hydrogen abstraction

$$
\mathrm{P}_{n}+\mathrm{R}^{\bullet} \rightarrow \mathrm{B}_{n}^{\bullet}+\mathrm{RH}
$$

Chain scission ( $\beta$-scission)

$$
\mathrm{B}_{n}^{\bullet} \rightarrow \mathrm{R}_{r}^{\bullet}+\mathrm{P}_{n-r}
$$

Chain end-radical transfer

$$
\mathrm{P}_{n}+\mathrm{R}_{r}^{\bullet} \rightarrow \mathrm{P}_{r}+\mathrm{B}_{n}^{\bullet}
$$

Termination by combination with primary radical

$$
\begin{aligned}
& \mathrm{R}^{\bullet}+\mathrm{B}_{n}^{\bullet} \rightarrow \mathrm{P}_{i} \\
& \mathrm{R}^{\bullet}+\mathrm{R}^{\bullet} \rightarrow \mathrm{P}_{i}
\end{aligned}
$$

Termination by disproportionation

$$
\mathrm{R}_{r}^{\bullet}+\mathrm{R}_{n}^{\bullet} \rightarrow \mathrm{P}_{r}+\mathrm{P}_{n}
$$

This scheme showed that chain scission is not instantaneous. A practical consequence of this assumption is the formation of radicals with active center located along the backbone $\left(\mathrm{B}^{\circ}\right)$ due to the abstraction of hydrogen. These radicals can undergo scission to generate polymer species with activity on the chain end $\left(\mathrm{P}^{\bullet}\right)$. It is also possible that combination between polymer radicals $\left(\mathrm{B}^{\bullet}\right.$ and $\left.\mathrm{P}^{\bullet}\right)$ and the primary radicals $\left(\mathrm{R}^{\circ}\right)$ occur, producing inactive polymer chains (P). The subscript $i, n$ and $r$ represent the chain lengths of the polymer species.

According to Simha-Wall model [32, 33], the mechanism of the thermal degradation of polypropene has been described as occurring by a random scission mechanism, in terms of first-order kinetics when the number of chain scissions is measured by the change in the degree of depolymerization. The main advantage of this model is the accurate description of the pyrolysis reaction in great detail. However, when random scission degradation is measured by weight loss, the kinetics will probably deviate from a first-order model $[32,46,47]$. By using apparatus such as TGA in kinetic studies, in fact the evaporation rate of products is determined and not the intrinsic chemical reaction rate, since not every broken bond leads to the evaporation of products but only product fragments which are small enough to evaporate will actually evaporate and thus lead to a decrease in the sample mass. Some authors proposed that zeroorder kinetic model is appropriate at low degree of conversion $(0<\alpha<0.2)$, but at higher degree of conversion $(0.7<\alpha<0.9)$ the more correct approach is to use first-order kinetic equation. As a consequence, the kinetic constants differ significantly over the conversion range $[46,48]$. According to Gao et al. [32, 33], it would be better to use fractional $n$-th order kinetic model to describe the pyrolysis kinetics of polypropene.

According to the random chain dissociation (RCD) model described in [46, 47], the pyrolysis process is a random process where each bond of the same type has an equal probability for cleavage, with a rate description according to a first order kinetic model (Figure 6).

Because of the differences in the stabilities of chains bonds caused by attached functional groups or radicals, a separate kinetic constant must be introduced for each type of bond. The model accounts for the fact that both physical and chemical processes play important roles during the pyrolysis of polymers. In this model, the mass of the polymer sample decreases due to evaporation of chains shorter than a certain 'evaporable' chain length. This parameter is a function of temperature and its value can be estimated from the boiling tem- 


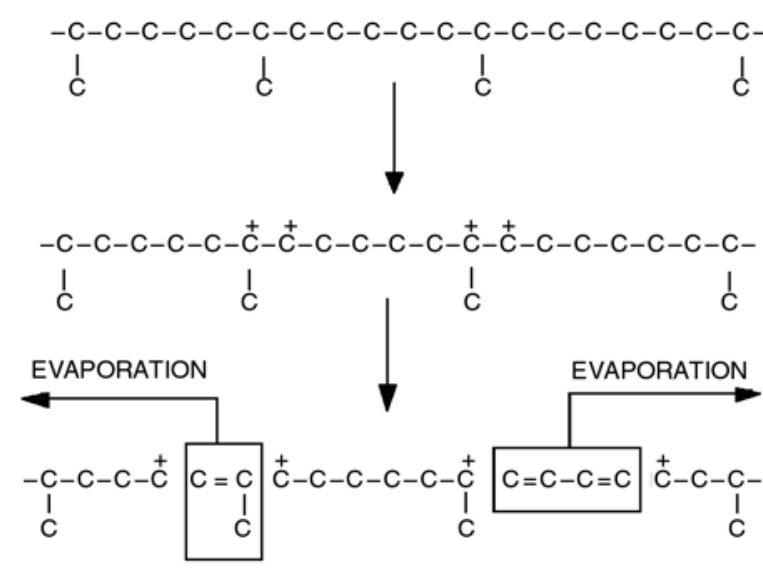

Figure 6. Schematic representation of pyrolysis process incorporated in the random chain dissociation (RCD) model

peratures of $n$-alkanes and $n$-alkenes. This approach implies that cleavage of the first bonds will not immediately result in evaporation of the degraded chains and, thus, this model predicts an initiation period with a comparatively low conversion rate. Following this initiation period, the conversion rate first increases until it reaches a maximum and then decreases due to a decrease of the available number of bonds. Due to the complex nature of this degradation mechanism of the polypropene, its kinetics is not possible to be described with zero, first or second order kinetics equations. By using apparatus as a TGA in kinetic studies, in fact the evaporation rate of products is determined and not the intrinsic chemical reaction rate, since not every broken bond in the polymer chain leads to the evaporation of products. Only product fragments which are small enough to evaporate will actually evaporate at given reaction temperature and thus lead to a decrease of the polymer mass [46]. This implies that both physical and chemical processes influence the measured rate of change of polymer mass and hence the observed pyrolysis kinetics. Incorporation of the influence of different types of chemical bonds and evaporation of the volatile products obtained through melted polypropene into the model complicate the establishing of the true kinetic mechanism of the pyrolysis process. Only computer simulations using different calculation procedures and kinetic equations (random nucleation, phase boundary reactions or diffusion) could possibly give the most probably kinetic parameters of the conversion process [2, 8, 49-51].
The thermal degradation of polypropene and polypropene composites begins in a melt with varying viscosity. Indeed, the presence of fillers increases significantly the viscosity of the molten polymer and filled polymer does not show Newtonian plateau at very low shear rate [52].

\section{Conclusions}

The thermal oxidative degradation kinetics of pure polypropene and polypropene filled with 20 mass\% different fillers was analyzed using Coats - Redfern, Madhysudanan et al., Tang et al., Wanjun et al. calculation procedures and 27 model kinetic equations. The kinetic parameters $E, A, \Delta S^{\neq}, \Delta H^{\neq}$ and $\Delta G^{\neq}$for all studied samples were calculated. The main results obtained in this article were summarized as follows.

(1) The results obtained on the basis of the four calculation procedures used were quite similar which implies that their average value could be taken.

(2) The kinetic of non-isothermal degradation of the samples studied was best described by kinetic equations of $n$-th order ( $F_{n}$ mechanism).

(3) Depending on the nature of the filler used, the values of $n$ varied from 0.66 to 1.63 .

(4) The highest values of $n, E$ and $A$ were observed for the composites filled with WRHA and Aerosil which have the same chemical composition $\left(\mathrm{SiO}_{2}\right)$ and porous structure.

(5) A linear dependence was found between $\ln A$ and $E$ of the samples studied, known also as kinetic compensation effect.

The results obtained were considered enough to conclude that the cheap raw rice husks (RRH) and the products of its thermal degradation (BRHA and WRHA), after vigorously grounding and mixing, cab successfully be used as fillers for polypropene to replace the expensive synthetic additive Aerosil in the preparation of different polypropene composites.

\section{References}

[1] Chen X., Yu B., Guo S.: Thermal oxidative degradation kinetics of $\mathrm{PP}$ and $\mathrm{PP} / \mathrm{Mg}(\mathrm{OH})_{2}$ flame-retarded composites. Journal of Applied Polymer Science, 103, 1978-1984 (2007) 
[2] Chen Y., Wang Q.: Thermal oxidative degradation kinetics of flame-retarded polypropylene with intumescent flame-retardant master batches in situ prepared in twin-screw extruder. Polymer Degradation and Stability, 92, 280-291 (2007).

[3] Wang L., Sheng J.: A kinetic study on the thermal degradation of polypropylene/attapulgite nanocomposites. Journal of Macromolecular Science, Part B: Physics, 45, 1-11 (2006).

[4] Zhang F., Zhang J., Wang Y.: Modeling study on the combustion of intumescent fire-retardant polypropylene. Express Polymer Letters, 1, 157-165 (2007).

[5] Marcilla A., Gómez A., Reyes-Labarta J. A., Giner A., Hernandez F.: Kinetic study of polypropylene pyrolysis using ZSM-5 and an equilibrium fluid catalytic cracking catalyst. Journal of Analytical and Applied Pyrolysis, 68-69, 467-480 (2003).

[6] Filho J. G. A. P., Graciliano E. C., Silva A. O. S., Souza M. J. B., Araujo A. S.: Thermo gravimetric kinetics of polypropylene degradation of ZSM-12 and ZSM-5 catalysts. Catalysis Today, 107-108, 507-512 (2005).

[7] Kim S-W., Cho Y-S., Shim M-J.: Thermal degradation kinetics of PE by the Kissinger equation. Materials Chemistry and Physics, 52, 94-97 (1998).

[8] Kim H. T., Oh S. C.: Kinetics of thermal degradation of waste polypropylene and high-density polyethylene. Journal of Industrial Engineering Chemistry, 11, 648-656 (2005).

[9] Gersten J., Fanberg V., Hetsroni G., Shindler Y.: Kinetic study of the thermal decomposition of polypropylene, oil shale, and their mixture. Fuel, 79, 1679-1686 (2000).

[10] Pehlivan H., Özmihçi F., Tihminlioglu T., Balköse D., Ülkü S.: Water and water wapor sorption studies in polypropylene-zeolite composites. Journal of Applied Polymer Science, 90, 3069-3075 (2003).

[11] Ismail H., Mohamad Z., Bakar A. A.: A comparative study of processing, mechanical properties, thermooxidative aging, water adsorption and morphology of rice husk powder and silica fillers in polystyrene/ styrene butadiene rubber blends. Polymer-Plastics Technology and Engineering, 42, 81-103 (2003).

[12] Kaci M., Djidjelli H., Boukerrou A., Zaidi L.: Effect of wood filler treatment and EBAGMA compatibilizer on morphology and mechanical properties of low density polyethylene/olive husk flour composites. Express Polymer Letters, 1, 467-473 (2007).

[13] Kim H. S., Yang H. S., Kim H. J., Park H. J.: Thermogravimetric analysis of rice husk flour filled thermoplastic polymer composites. Journal of Thermal Analysis and Calorymetry, 76, 395-404 (2004).

[14] Razavi-Nouri M., Jafarzadeh-Dogouri F., Oromiehie A. R., Langroudi A. E.: Mechanical properties and water adsorption behaviour of chopped rice husk filled polypropylene composites. Iranian Polymer Journal, 15, 757-766 (2006).
[15] Chaudhary D. S., Jollands M. C., Cser F.: Understanding rice hull ash as fillers in polymers: A review. Silicon Chemistry, 1, 281-289 (2002).

[16] Panthapulakkal S., Law S., Sain M.: Enhancement of processability of rice husk filled high-density polyethylene composite profiles. Journal of Thermoplastics Composites Materials, 18, 445-458 (2005).

[17] Ballice L., Reimert R.: Classification of volatile products from the temperature-programmed pyrolysis of polypropylene (PP), atactic-polypropylene (APP) and thermogravimetrically derived kinetics of pyrolysis. Chemical Engineering Processing, 41, 289-296 (2002).

[18] Durmuş A., Koç S. N., Pozan G. S., Kaşgöz A.: Thermal-catalytic degradation kinetics of polypropylene over BEA, ZSM-5 and MOR zeolites. Applied Catalysis, Part B: Environmental, 61, 316-322 (2005).

[19] Peterson D. J., Vyazovkin S., Wight C. A.: Kinetics of the thermal and thermo-oxidative degradation of polystirene, polyethylene and poly(propylene). Macromolecular Chemistry and Physics, 202, 775-784 (2001).

[20] Song R., Jiang Z., Bi W., Cheng W., Lu J., Huang B., Tang T.: The combinated catalytic action on solid acids with nickel for the transformation of polypropylene into carbon nanotubes by pyrolysis. Chemistry-A European Journal, 13, 3234-3240 (2007).

[21] Kruse T. M., Wong H-W., Broadbelt L. J.: Mechanistic modeling of polymer pyrolysis: polypropylene. Macromolecules, 6, 9594-9607 (2003).

[22] Dawood A., Miura K.: Pyrolysis kinetics of $\gamma$-irradiated polypropylene. Polymer Degradation and Stability, 73, 347-354 (2001).

[23] Carniti P., Gervasini A.: Thermogravimetric study of the kinetics of degradation of polypropylene with solid catalysts. Thermochimica Acta, 379, 51-58 (2001).

[24] Tihminlioglu F., Pehlivan H., Balköse D., Ülkü S.: Effect of the zeolite filler on the thermal degradation kinetics of polypropylene. Oxidation Communications, 29, 193-203 (2006).

[25] Marcilla A., Gomez A., Reyes-Labarta J. A., Giner A.: Catalytic pyrolysis of polypropylene using MCM-41: Kinetic model. Polymer Degradation and Stability, 80, 233-240 (2003).

[26] Ramis X., Cadenato A., Salla J. M., Morancho J. M., Valles A., Contat L., Ribes A.: Thermal degradation of polypropylene/starch-based materials with enhanced biodegradability. Polymer Degradation and Stability, 86, 483-491 (2004).

[27] Vlaev L. T., Markovska I. G., Lyubchev L. A.: Nonisothermal kinetics of pyrolysis of rice husk. Thermochimica Acta, 406, 1-7 (2003).

[28] Albano C. L., Sciamanna E. S., Aquno T., Martinez J. J.: Metodology to evaluate thermogravimetric data using computational techniques in the polymer field. European Congress on Computational Methods in Applied Science and Engineering, ECCOMAS 2000, Barcelona, Spain 11-14 Sept., pp. 1-18 (2000). 
[29] Roy P. K., Surekha P., Rajagopal C., Choudhary V.: Thermal degradation studies of LDPE containing cobalt stearate as pro-oxidant. Express Polymer Letters, 1, 208-216 (2007).

[30] Vlaev L., Georgieva V., Genieva S.: Products and kinetics of non-isothermal decomposition of vanadium(IV) oxide compounds. Journal of Thermal Analysis and Calorymetry, 88, 805-812 (2007).

[31] Šestak J., Berggren G.: Study of the kinetics of the mechanism of solid-state reactions at increasing temperatures. Thermochimica Acta, 3, 1-12 (1971).

[32] Gao Z., Kaneko T., Amasaki I., Nakada M.: A kinetic study of thermal degradation of polypropylene. Polymer Degradation and Stability, 80, 269-274 (2003).

[33] Gao Z., Amasaki I., Nakada M.: A thermogravimetric study on thermal degradation of polyethylene. Journal of Analytical and Applied Pyrolysis, 67, 1-9 (2003).

[34] Coats W., Redfern J. P.: Kinetic parameters for thermogravimetric data. Nature, 201, 68-69 (1964).

[35] Madhysudanan P. M., Krishnan K., Ninan K. N.: New equations for kinetic analysis of non-isothermal reactions. Thermochimica Acta, 221, 13-21 (1993).

[36] Tang W., Liu Y., Zang H., Wang C.: New approximate formula for Arrhenius temperature integral. Thermochimica Acta, 408, 39-43 (2003).

[37] Wanjun T., Yuwen L., Hen Z., Zhiyong W., Cunxin W.: New temperature integral approximate formula for non-isothermal kinetic analysis. Journal of Thermal Analysis and Calolimetry, 74, 309-315 (2003).

[38] Ruvolo-Filho A., Curti P. S.: Chemical kinetic model and thermodynamic compensation effect of alkaline hydrolysis of waste poly(ethylene terepftalate) in nonaqueous ethylene glycol solution. Industrial Engineering Chemistry Research, 45, 7985-7996 (2006).

[39] Nikolaev A. V., Logvinenko V. A., Gorbatchov V. M., Miachina L. I.: Thermal analysis. in 'Proceedings of the Fourth ICTA, Budapest Hungary' vol. 1, 47 (1974).

[40] Zmijewski T., Pysiak J.: Thermal analysis. in 'Proceedings of the Fourth ICTA, Budapest Hungary' vol. 1, 205 (1974).

[41] Vlaev L. T., Georgieva V. G., Genieva S. D.: Use of the ion polarization theory to interpret certain regularities of changes in characteristics and properties of inorganic compounds. Journal of Structural Chemistry, 47, 813-822 (2006).

[42] Nakatani H., Suzuki S., Tanaka T., Terano M.: New kinetic aspects on the mechanism of thermal oxidative degradation of polypropylenes with various tacticities. Polymer, 46, 12366-12371 (2005).
[43] Oliveira J. A., Biscaia E. C. Jr., Pinto J. C.: Analysis of kinetic models proposed for the controlled degradation of poly(propylene)-presentation of a general and analytical solution. Macromolecular Theory and Simulation, 12, 696-704 (2003).

[44] Bockhorn H., Hornung A., Hornung U., Schawaller D.: Kinetic study on the thermal degradation of polypropylene and polyethylene. Journal of Analysis and Applied Pyrolysis, 48, 93-109 (1999).

[45] Chan J. H., Balke S. T.: The thermal degradation kinetics of polypropylene: Part I. Molecular weight distribution. Polymer Degradation and Stability, 57, 113-125 (1997).

[46] Westerhout R. M. J., Waanders J., Kuipers J. A. M., van Swaaij W. P. M.: Kinetics of the low-temperature pyrolysis of polyethene, polypropene, and polystyrene modeling, experimental, determination, and comparison with literature podels and data. Industrial and Engineering Chemistry Research, 36, 1955-1964 (1997).

[47] Westerhout R. M. J., Balk R. H. P., Meijer R., Kuipers J. A. M., van Swaaij W. P. M.: Experimentation and evaluation of the use of screen heaters for the measurement of the high temperature pyrolysis kinetics of polyethene and polypropene. Industrial and Engineering Chemistry Research, 36, 3360-3368 (1997).

[48] Qin H., Zhang S., Zhao C., Yang M.: Zero-order kinetics of the thermal degradation of polypropylene/ clay nanocomposites. Journal of Polymer Science: Part B: Polymer Physics, 43, 3713-3719 (2005).

[49] Contat-Rodrigo L., Ribes-Greus A., Díaz-Calleja R.: Characterization by thermal analysis of PP with enhanced biodegradability. Journal of Applied Polymer Science, 82, 2174-2184 (2001).

[50] Chan J. H., Balke S. T.: The thermal degradation kinetics of polypropylene: Part II. Time-temperature superposition. Polymer Degradation and Stability, 57, 127-134 (1997).

[51] Chan J. H., Balke S. T.: The thermal degradation kinetics of polypropylene: Part III. Thermogravimetric analyses. Polymer Degradation and Stability, 57, 135-149 (1997).

[52] La Mantia F. P., Dintcheva N. T.: Thermomechanical degradation of filled polypropylene. Macromolecular Symposia, 194, 277-286 (2003). 